\title{
Lymph node stroma join the cancer support network
}

\author{
Anne L Fletcher ${ }^{*, 1}$ and Tracy SP Heng ${ }^{*, 2}$ \\ Cell Death and Differentiation (2016) 23, 1899-1901; doi:10.1038/cdd.2016.103; published online 30 September 2016
}

Lymph nodes (LNs) are sentinels of the immune system. They allow the lymph draining from peripheral tissues to pass through a leucocytic filter, bringing lymph-borne antigens, inflammatory mediators and antigen presenting cells into close proximity with immune cells capable of responding to antigen exposure. ${ }^{1}$ Although LNs are often the first site of tumour metastasis for many cancers, it remains unclear how tumour development affects LN structure and function, and how these changes in turn drive tumour growth. Riedel et al. ${ }^{2}$ have now shown that tumour-draining lymph nodes (TDLNs) respond dynamically to tumour-derived signals early in tumour development, with transcriptional, compositional and structural changes that have important implications for tumour growth.

The structure of the LN is created by fibroblastic reticular cells (FRCs), immunologically specialised stromal cells of mesenchymal origin that form cellular and extracellular networks, providing structural support and chemical cues essential for immune function. ${ }^{3}$ Notably, FRCs produce chemokines and cytokines for immune cell survival and trafficking within LNs. ${ }^{4,5}$ They also create and ensheathe ultrafine conduits that permit rapid dissemination of fluid and small molecules, such as chemokines, cytokines and soluble antigens, throughout the parenchyma. ${ }^{6}$

During an infection, cellular interactions generally lead to priming of antigen-specific $T$ cells and the initiation of a robust immune response against the infectious agent. In TDLNs, however, the stimulation of anti-tumour responses is often hampered by the induction of immune suppression. ${ }^{7}$ Ineffective anti-tumour immunity within TDLNs can lead to the spread of malignant cells to distal organs. Despite important roles for FRCs in fostering healthy, efficient immunological function, little is known about how they respond to the influx of tumourassociated inflammatory mediators, cells and antigens.

To address this question, Riedel et al. ${ }^{2}$ compared FRCs from TDLNs and non-draining LNs, utilising two different mouse models of melanoma at a pre-metastatic stage. They showed that TDLN FRCs undergo transcriptional changes and mediate structural remodelling to generate an altered microenvironment hypothesised to be tumour conducive. These pre-metastatic LNs increased in size, caused by an increase in leucocytes, followed by proliferation of FRCs, thought to provide greater support for enlarging conduits (Figure 1).

Using microarray analysis, Riedel et al. showed that FRCs underwent transcriptional reprogramming of several signalling pathways as tumours developed upstream of TDLNs. Principal component analysis revealed that the majority of transcriptional differences were acquired before day 4, when tumours were hardly palpable (PC1: $88.9 \%$ of variance), with a much smaller degree of change occurring between day 4 and day 11 when tumours were established (PC2: $4.5 \%$ of variance). This suggests that major changes to FRCs occur early, during a period accompanied by significant TDLN expansion. This is in accordance with studies showing that an increase in haematopoietic cells almost immediately induces FRC cytoskeletal remodelling to accommodate the LN's expanding size. ${ }^{8,9}$ The relatively low level of transcriptional difference between day 4 and day 11 would suggest that upon the first palpable sign of tumour infiltration, the LN had already undergone substantial transcriptional changes to become activated and, perhaps, more conducive to tumour development, although functional studies are yet to specifically assess this.

The lack of a distinctive 'TDLN-FRC' signature was a surprising finding, and may be a product of the early time points necessitated by the metastatic aggression of the models used. Of 117 genes upregulated at day 4, only 25 genes were still upregulated by day 11; and of 106 genes downregulated at day 4,39 were still downregulated by day 11 . Whether tumouractivated FRCs develop a distinct, stable phenotype in the presence of tumour growth over a longer timescale remains to be explored in a slower-growing, non-metastatic model.

Genes relevant to proliferation, metabolism, mitochondrial dysfunction, movement and migration, and cell-junction pathways were upregulated by FRCs in TDLNs compared with non-draining LNs, whereas those encoding cytokines and chemokines for leucocyte trafficking and survival, for example, CCL21 and IL-7, were downregulated (Figure 1). Accordingly, changes in the composition and localisation of immune cell types within the TDLN were observed. Decreased IL-7 and CCL21 expression by TDLN FRCs correlated with a small but statistically significant reduction in the proportion, but presumably not number, of $\mathrm{CD}^{+} \mathrm{T}$ cells, and a blurring of the typically distinct $\mathrm{T}$ and $\mathrm{B}$ cell boundaries. Upregulation of

\footnotetext{
${ }^{1}$ Institute of Immunology and Immunotherapy, University of Birmingham, Birmingham B15 2T, UK and ${ }^{2}$ Biomedicine Discovery Institute, and Department of Anatomy and Developmental Biology, Monash University, Clayton 3800, VIC, Australia

${ }^{*}$ Corresponding author: AL Fletcher, Institute of Immunology and Immunotherapy, College of Medical and Dental Sciences, University of Birmingham, Edgbaston B15 2TT, UK. Tel: +44 121414 2268; Fax: +44 (0)121 414 3599; E-mail: A.Fletcher@bham.ac.uk

or TSP Heng, Department of Anatomy and Developmental Biology, Monash University, Level 3, 15 Innovation Walk, Clayton 3800, VIC, Australia. Tel: +61 399050629 ; E-mail: Tracy.Heng@monash.edu
} 


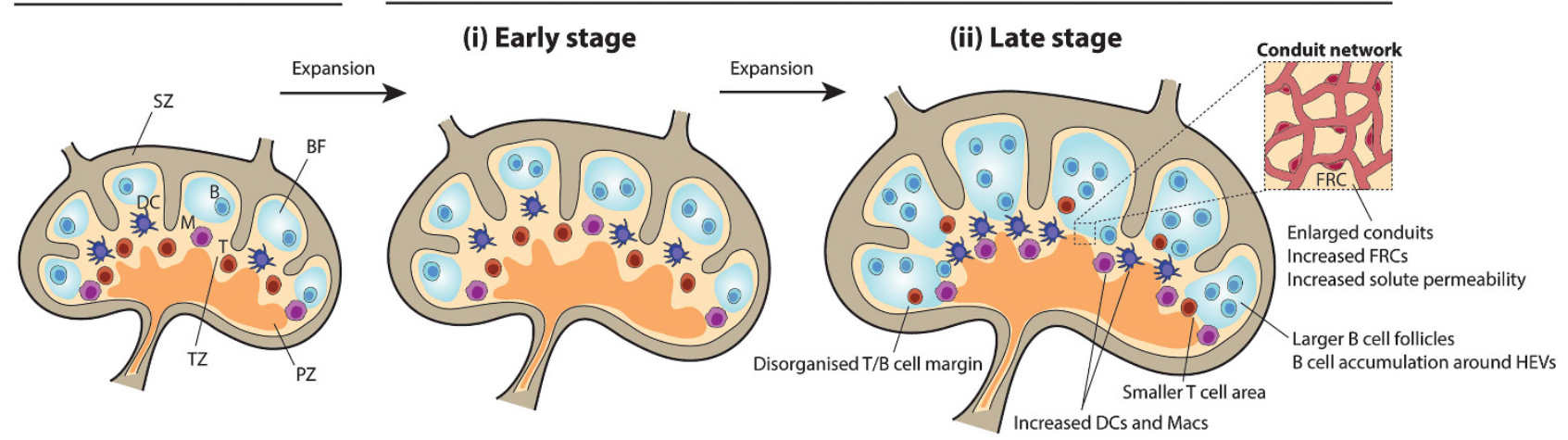

GENE EXPRESSION CHANGES

\begin{tabular}{|c|c|c|}
\hline $\begin{array}{l}\text { Proliferation, Mitochondrial Dysfunction, Metabolism, } \\
\text { Junction Molecules, Movement and Migration }\end{array}$ & $\uparrow$ & $\uparrow \uparrow$ \\
\hline Signalling Molecules (e.g. Limk2, Kras, Tgfbr2) & $\uparrow$ & $\uparrow \uparrow$ \\
\hline Cytokines and Chemokines (e.g. II7, Cc/21) & $\downarrow$ & $\downarrow \downarrow$ \\
\hline Fibroblast Activation Markers (e.g. Pdpn, S100a4, Thy1, Cd248) & $\uparrow$ & $\uparrow \uparrow$ \\
\hline Channels and Ion Transporters (e.g. Aqp1) & $\uparrow \uparrow$ & $\uparrow$ \\
\hline
\end{tabular}

Figure 1 Transcriptional, structural and compositional changes in the LN stroma in response to tumour-derived cues. Compared with a non-draining LN (a), the TDLN undergoes a transitional early stage of transformation (b(i)), expanding in size and increasing in FRC numbers. During the late stage of transformation (b(ii)), enlarged conduits allow for increased permeability of solutes, whereas changes in composition and localisation of immune cells are accompanied by disorganisation of a normally distinct T/B cell margin. Selected dysregulated genes are categorised by general function, and changes in the TDLN during early and late stages of tumour growth are indicated as upregulated $(\uparrow)$ or downregulated $(\downarrow)$

monocyte/dendritic cell chemotactic factors was in line with an increased proportion of dendritic cells and macrophages. $\mathrm{T}$ cells in TDLNs were still becoming activated, but $\mathrm{CD}^{+}$ $T$ cells exhibited decreased homing efficiency into the $L N$, whereas B cells showed no inward homing deficiencies, but appeared less able to migrate out of the LN, accumulating instead around high endothelial venules.

The authors indicated that the structural disorganisation in TDLNs may be reminiscent of plt/plt mice, which lack CCL19 and CCL21. ${ }^{10} \mathrm{Plt} / \mathrm{plt}$ mice show $\mathrm{T}$ cell responses that are delayed in initiation but ultimately increased in magnitude, ${ }^{10}$ so it would be interesting to directly ascertain the impact of the structural disorganisation observed in TDLNs on anti-tumour T cell function.

Overall, TDLN FRCs underwent transcriptional changes that indicated activation and perhaps a pro-fibrotic 'cancerassociated fibroblasts (CAF)-like' state, upregulating fibroblast activation markers and displaying increased granularity. The direct link between exposure to tumour-derived factors and fibroblast activation was supported by treating FRCs with tumour-conditioned medium, which led to upregulation of podoplanin and increased capacity of cultured FRCs to contract collagen gel. The authors suggest that wider conduits and increased collagen production may induce increased TDLN stiffness, and at least one other study of B16 melanoma at similar time points has demonstrated an increase in elasticity in TDLNs, similarly accompanied by increased collagen production and intranodal pressure. ${ }^{11}$ The relative contribution of mechanical and chemical stimuli to the transcriptional changes observed by Riedel et al. would be fascinating to address.
Additionally, an FRC monolayer treated with tumourconditioned medium permitted the passage of high molecular weight dextran in transwell experiments, suggesting that soluble tumour-derived factors might encounter a less selective barrier and gain access into TDLNs. This increased permeability was confirmed by confocal microscopy showing greater penetration of dextran into the paracortical areas of the TDLN.

These changes in the metastatic melanoma model induced by subcutaneous injection of B16.F10 tumour cells were similarly observed in the $\mathrm{Tyn}^{\mathrm{CreER}}{ }^{\mathrm{Braf}}{ }^{\mathrm{CA}} \mathrm{Pten}^{\text {lox }}$ genetic mouse model of metastatic melanoma (albeit with larger data spread), excluding the possibility that the observed changes were induced by pathogen-driven inflammation. Accordingly, genes key to leukocyte migration and survival, notably IL-7 and CCL21, were downregulated in TDLN, in opposition to at least one inflammatory model where IL-7 was upregulated and CCL21 remained unchanged, ${ }^{12}$ albeit at a $12 \mathrm{~h}$ time point and before significant LN expansion, making direct comparison with the day 4 or day 11 data difficult.

This study by Riedel et al. is the first to comprehensively profile transcriptional changes to the FRC compartment within TDLNs. These data sets will provide a valuable resource to the immunological community for further interrogation and study. It would be most interesting now to test whether these FRC-induced changes in immune cell composition, homing and function translate, as predicted, to a clinically relevant functional outcome (e.g. increased immunosuppression or tumour promotion), to definitively prove that FRC reprogramming directly drives a suppressive, pro-tumour environment. Such a study would also be highly relevant to the 
long-standing debate regarding routine surgical excision of TDLNs, which may help or hinder anti-tumour immunity depending on the ability of FRCs and other cells to influence with the generation of effective immune responses. Animal studies examining the effect of $L N$ removal before tumour inoculation would also be informative. Lastly, a comparative exploration of FRCs and the FRC-like CAFs, which develop within or surrounding tumours would be of immediate interest to the field, since CAFs and tumour-draining FRCs share many surface markers and are each likely to impose direct immunosuppressive effects.

\section{Conflict of Interest}

The authors declare no conflict of interest.

Acknowledgements. We thank Jorge Caamano, Sophie Acton, Jillian Astarita, Deepali Malhotra and Daniel Gray for helpful discussion. TH is supported by an R.D.
Wright Career Development Fellowship from the National Health and Medical Research Council (APP1107188), a Linkage Project grant from the Australian Research Council (LP110201169) and the National Stem Cell Foundation of Australia. AF is supported by a Birmingham Fellowship and a Wellcome Trust Seed Award.

\footnotetext{
1. Fletcher AL, Acton SE, Knoblich K. Nat Rev Immunol 2015; 15: 350-361.

2. Riedel A et al. Nat Immunol 2016.

3. Bajenoff M et al. Immunity 2006; 25: 989-1001.

4. Link A et al. Nat Immunol 2007; 8: 1255-1265.

5. Katakai T et al. J Exp Med 2004; 200: 783-795.

6. Gretz JE et al. J Exp Med 2000; 192: 1425-1440.

7. Fransen MF, Arens R, Melief CJ. Int J Cancer 2013; 132: 1971-1976.

8. Acton SE et al. Nature 2014; 514: 498-502.

9. Astarita JL et al. Nat Immunol 2015; 16: 75-84.

10. Mori S et al. J Exp Med 2001; 193: 207-218.

11. Rohner NA et al. FASEB J 2015; 29: 4512-4522.

12. Malhotra D et al. Nat Immunol 2012; 13: 499-510.
} 